\title{
Kernel Decompositions for Schur Functions on the Polydisk
}

\author{
Greg Knese
}

Received: 7 September 2009 / Accepted: 27 January 2010 / Published online: 12 February 2010

(C) The Author(s) 2010. This article is published with open access at Springerlink.com

\begin{abstract}
A certain kernel (sometimes called the Pick kernel) associated to Schur functions on the disk is always positive semi-definite. A generalization of this fact is well-known for Schur functions on the polydisk. In this article, we show that the "Pick kernel" on the polydisk has a great deal of structure beyond being positive semidefinite. It can always be split into two kernels possessing certain shift invariance properties.
\end{abstract}

Keywords Schur function · Polydisk · Polydisc · Reproducing kernel · Agler decomposition · Pick interpolation

Mathematics Subject Classification (2000) Primary 47A57; Secondary 42B05

\section{Introduction}

Let $\mathbb{D}^{d}$ be the unit polydisk in $\mathbb{C}^{d}$. A Schur function is simply a holomorphic function $f: \mathbb{D}^{d} \rightarrow \mathbb{C}$ bounded by one in modulus. One of the most fundamental facts about Schur functions in one variable is that the following kernel is positive semi-definite:

$$
\frac{1-f(z) \overline{f(\zeta)}}{1-z \bar{\zeta}} \geq 0
$$

Communicated by Mihai Putinar.

G. Knese ( $\varangle)$

University of California, Irvine, Irvine, CA 92697-3875, USA

e-mail: gknese@uci.edu 
(we say a function $K: \mathbb{D}^{d} \times \mathbb{D}^{d} \rightarrow \mathbb{C}$ is a positive semi-definite kernel and write $K \geq 0$ if for every finite subset $F \subset \mathbb{D}^{d}$, the matrix

$$
(K(z, \zeta))_{z, \zeta \in F}
$$

is positive semi-definite - to actually form a matrix we would need an ordering of $F$, but this is unimportant).

The positive semi-definiteness of (1.1) is significant because (1) it relates function theory to operator theory and (2) it turns out to have a very strong converse: if $f$ is a function on a finite subset of $\mathbb{D}$ such that (1.1) is positive semi-definite on that finite set, then $f$ is the restriction of a Schur function. This is the content of the Pick interpolation theorem.

It is not clear what the "best" generalization of (1.1) is to several variables. For a Schur function in $d$ variables, it is a fact that

$$
\frac{1-f(z) \overline{f(\zeta)}}{\prod_{j=1}^{d}\left(1-z_{j} \bar{\zeta}_{j}\right)}
$$

is positive semi-definite, however this does not seem to be extremely useful. Here $z=\left(z_{1}, \ldots, z_{d}\right), \zeta=\left(\zeta_{1}, \ldots, \zeta_{d}\right) \in \mathbb{C}^{d}$.

It was not until ca. 1988 that a more useful result was given in two variables by Agler [1]: for any Schur function $f$ on $\mathbb{D}^{2}$ there exist positive semi-definite kernels $\Gamma_{1}, \Gamma_{2}: \mathbb{D}^{2} \times \mathbb{D}^{2} \rightarrow \mathbb{C}$ such that

$$
1-f(z) \overline{f(\zeta)}=\left(1-z_{1} \bar{\zeta}_{1}\right) \Gamma_{1}(z, \zeta)+\left(1-z_{2} \bar{\zeta}_{2}\right) \Gamma_{2}(z, \zeta)
$$

This formula, called an Agler decomposition, does not generalize to more variables in the way that its form suggests. Schur functions which satisfy

$$
1-f(z) \overline{f(\zeta)}=\sum_{j=1}^{d}\left(1-z_{j} \bar{\zeta}_{j}\right) \Gamma_{j}(z, \zeta)
$$

for some positive semi-definite kernels $\Gamma_{1}, \ldots, \Gamma_{d}$, form a proper subclass of the set of Schur functions called the Schur-Agler class.

Very recently, Grinshpan et al. [4] proved a decomposition that does hold in general and which is still analogous to (1.3). We state it here in the scalar valued case (in [4] it was proved in the operator-valued case).

Theorem 1.1 (Grinshpan et al. [4]) Let $f: \mathbb{D}^{d} \rightarrow \mathbb{D}$ be holomorphic. Then, for each $j, k \in\{1, \ldots, d\}: j \neq k$ there exist positive semi-definite kernels $K$ and $K^{\prime}$ such that

$$
1-f(z) \overline{f(\zeta)}=\prod_{r \neq j}\left(1-z_{r} \bar{\zeta}_{r}\right) K(z, \zeta)+\prod_{r \neq k}\left(1-z_{r} \bar{\zeta}_{r}\right) K^{\prime}(z, \zeta)
$$


It is our goal to strengthen this theorem and to alter the point of view slightly. Rather than looking for more decompositions analogous to (1.3), we instead attempt to illuminate the structure of the kernel in (1.2).

Before presenting our theorem we need the following definition.

Definition 1.2 If $K$ is a positive semi-definite kernel on $\mathbb{D}^{d}$, we shall say $K$ is $z_{j}$-contractive if

$$
\left(1-z_{j} \bar{\zeta}_{j}\right) K(z, \zeta) \geq 0
$$

If $S \subset\{1, \ldots, d\}$, then we say a kernel $K$ is $S$-contractive if it is $z_{j}$-contractive for all $j \in S$.

Theorem 1.3 Let $d \geq 2$ and let $f: \mathbb{D}^{d} \rightarrow \mathbb{D}$ be holomorphic. Then, for each non-empty $S \subsetneq\{1,2, \ldots, d\}$, there exist positive semi-definite $S$-contractive kernels $K_{S}, L_{S}$, such that if $S \sqcup T=\{1, \ldots, d\}$ is a non-trivial partition, then

$$
\begin{aligned}
\frac{1-f(z) \overline{f(\zeta)}}{\prod_{j=1}^{d}\left(1-z_{j} \bar{\zeta}_{j}\right)} & =K_{S}(z, \zeta)+L_{T}(z, \zeta) \\
K_{T}-L_{T} & =K_{S}-L_{S} \geq 0
\end{aligned}
$$

and if $S \subset S^{\prime} \subset\{1,2, \ldots, d\}$ then

$$
K_{S} \geq K_{S^{\prime}}
$$

Kernel inequalities like the last line should be interpreted as saying $K_{S}-K_{S^{\prime}}$ is positive semi-definite.

The proof of Theorem 1.1 in [4] amounts to the case where $S$ is a singleton, however many of the decompositions provided by Theorem 1.3 can be used to reprove Theorem 1.1.

Indeed, let $S \sqcup T=\{1, \ldots, d\}$ be any partition with $j \in S$ and $k \in T$. Theorem 1.1 follows from writing as in Theorem 1.3

$$
\begin{aligned}
1-f(z) \overline{f(\zeta)}= & \prod_{r \neq j}\left(1-z_{r} \bar{\zeta}_{r}\right)(\underbrace{\left.\left(1-z_{j} \bar{\zeta}_{j}\right) K_{S}(z, \zeta)\right)}_{K(z, \zeta)} \\
& +\prod_{r \neq k}\left(1-z_{r} \bar{\zeta}_{r}\right)(\underbrace{\left.\left(1-z_{k} \bar{\zeta}_{k}\right) L_{T}(z, \zeta)\right)}_{K^{\prime}(z, \zeta)}
\end{aligned}
$$

and $K$ and $K^{\prime}$ are positive since $K_{S}$ is $z_{j}$-contractive and $L_{T}$ is $z_{k}$-contractive.

Our proof of Theorem 1.3 relies on proving the result first for rational inner functions continuous on $\overline{\mathbb{D}^{d}}$; these can be characterized as follows.

Let $p \in \mathbb{C}[z]=\mathbb{C}\left[z_{1}, \ldots, z_{d}\right]$ have no zeros on the closed polydisk $\overline{\mathbb{D}^{d}}$ and suppose $\operatorname{deg} p \leq n=\left(n_{1}, \ldots, n_{d}\right)$. Define 


$$
\tilde{p}(z):=z^{n} \overline{p(1 / \bar{z})}=z_{1}^{n_{1}} \cdots z_{d}^{n_{d}} \overline{p\left(1 / \overline{z_{1}}, \ldots, 1 / \overline{z_{d}}\right)}
$$

(and notice $|p|=|\tilde{p}|$ on the $d$-torus $\mathbb{T}^{d}$ ).

Every regular rational inner function can be represented as $f(z)=\tilde{p}(z) / p(z)$ for some choice of $p$ and some choice of $n \geq \operatorname{deg}(p)$ as above (see Rudin [5] Theorem 5.2.5). We state a theorem below describing the structure of the following kernel

$$
\mathcal{P}(z, \zeta):=\frac{p(z) \overline{p(\zeta)}-\tilde{p}(z) \overline{\tilde{p}(\zeta)}}{\prod_{j=1}^{d}\left(1-z_{j} \bar{\zeta}_{j}\right)}
$$

a trivial modification of (1.2) in the case of $f=\tilde{p} / p$.

First, we need another definition.

Definition 1.4 Let us call $K(z, \zeta): \mathbb{D}^{d} \times \mathbb{D}^{d} \rightarrow \mathbb{C}$ a $\mathcal{P}$-kernel if

- $\mathcal{P} \geq K \geq 0$ in the sense of kernels and

- whenever $\mathcal{P}(z, \zeta) \geq f(z) \overline{f(\zeta)}$ and $K(z, \zeta) \geq \epsilon f(z) \overline{f(\zeta)}$ for some $\epsilon>0$, then we necessarily have $K(z, \zeta) \geq f(z) \overline{f(\zeta)}$.

See Lemma 7.5 in the Appendix for a precise description of what this means. The (aesthetic) point here is that we have a theorem which does not refer to our methods of proof. The follow theorem is similar to Theorem 1.3 but more precise.

Theorem 1.5 Let $p \in \mathbb{C}[z]$ be as above. For every non-empty $S \subsetneq\{1,2, \ldots, d\}$, there exist $S$-contractive $\mathcal{P}$-kernels $K_{S}, L_{S}$, such that if $S \sqcup T=\{1, \ldots, d\}$ is a non-trivial partition, then

$$
\mathcal{P}=K_{S}+L_{T}
$$

Moreover, $K_{S}$ is maximal among all $S$-contractive kernels bounded above by $\mathcal{P}$.

This last condition makes these decompositions unique.

\section{The Kernel $\mathcal{P}$}

The theorems from the introduction are proved by analyzing orthogonality relations for a "Bernstein-Szegô measure":

$$
d \mu=\frac{1}{|p(z)|^{2}} d \sigma(z)
$$

where $d \sigma$ is normalized Lebesgue measure on the $d$-torus $\mathbb{T}^{d}$ and $p \in \mathbb{C}[z]$ has no zeros on the closed polydisk $\overline{\mathbb{D}^{d}}$. We also use $d \sigma$ to represent normalized Lebesgue measure on different dimensional tori, and the dimension will be made apparent by the variable; e.g. $d \sigma\left(z_{1}\right)$ corresponds to normalized Lebesgue measure on $\mathbb{T}$ using the variable $z_{1}$. 
Notice that the complex Hilbert space $L^{2}(\mu)$ is a renorming of $L^{2}\left(\mathbb{T}^{d}\right)$ and therefore is topologically isomorphic. The inner product on $L^{2}(\mu)$ is denoted

$$
\langle f, g\rangle_{\mu}=\int_{\mathbb{T}^{d}} f(z) \overline{g(z)} d \mu(z) .
$$

For a subset $X$ of the lattice $\mathbb{Z}^{d}$ we define the closed subspace

$$
L_{\mu}^{2}(X):=\left\{f \in L^{2}(\mu): \hat{f}(\alpha)=0 \text { for } \alpha \notin X\right\}
$$

where $\hat{f}(\alpha)$ denotes the $\alpha$ th Fourier coefficient of $f$ (and note we typically use $\alpha=\left(\alpha_{1}, \ldots, \alpha_{d}\right)$ to denote a $d$-tuple of integers). We use the following non-traditional notation. If $Y \subset X \subset \mathbb{Z}^{d}$ then we write

$$
L_{\mu}^{2}(X \ominus Y):=L_{\mu}^{2}(X) \ominus L_{\mu}^{2}(Y)
$$

We use the following partial order on $d$-tuples of integers $\alpha=\left(\alpha_{1}, \ldots, \alpha_{d}\right)$, $\beta=\left(\beta_{1}, \ldots, \beta_{d}\right)$ :

$$
\alpha \leq \beta \text { if and only if } \alpha_{j} \leq \beta_{j} \text { for all } j=1, \ldots, d
$$

$n=\left(n_{1}, \ldots, n_{d}\right)$ is a fixed $d$-tuple which bounds the multi-degree of $p$ (i.e. the degree of $p$ with respect to $z_{j}$ is at most $n_{j}$ ); writing $\alpha<\beta$ means $\alpha \leq \beta$ and $\alpha \neq \beta$.

We typically write elements of $\mathbb{C}^{d}$ with $z=\left(z_{1}, \ldots, z_{d}\right)$. We use multi-index notation:

$$
z^{\alpha}:=z_{1}^{\alpha_{1}} \cdots z_{d}^{\alpha_{d}}
$$

for $\alpha \in \mathbb{Z}^{d}$ and $z \in \mathbb{C}^{d}$.

We need to define various subsets of $\mathbb{Z}^{d}$ :

$$
\begin{aligned}
\mathbb{Z}_{+}^{d} & :=\left\{\alpha \in \mathbb{Z}^{d}: \alpha \geq 0\right\} \\
\mathbb{Z}_{n+}^{d} & :=\left\{\alpha \in \mathbb{Z}^{d}: \alpha \geq n\right\} \\
B & :=\mathbb{Z}_{+}^{d} \backslash \mathbb{Z}_{n+}^{d}=\left\{\alpha \in \mathbb{Z}_{+}^{d}: \exists j: \alpha_{j}<n_{j}\right\}=\left\{\alpha \in \mathbb{Z}_{+}^{d}: \alpha \nsupseteq n\right\}
\end{aligned}
$$

Then, for example $L_{\mu}^{2}\left(\mathbb{Z}_{+}^{d}\right)$ denotes the closure of the polynomials with respect to $L^{2}(\mu)$, a space equal to the Hardy space $H^{2}\left(\mathbb{T}^{d}\right)$ although it has a different inner product.

The first thing we prove provides the connection to the kernel $\mathcal{P}$. See [2] for background on reproducing kernel Hilbert spaces. The Szegö kernel will be denoted:

$$
\mathcal{S}_{d}(z, \zeta)=\prod_{j=1}^{d} \frac{1}{\left(1-z_{j} \bar{\zeta}_{j}\right)}
$$


As $H^{2}\left(\mathbb{T}^{d}\right)$ is a reproducing kernel Hilbert space with kernel $\mathcal{S}_{d}$ and since $L_{\mu}^{2}\left(\mathbb{Z}_{+}^{d}\right)$ is a renorming of $H^{2}\left(\mathbb{T}^{d}\right), L_{\mu}^{2}\left(\mathbb{Z}_{+}^{d}\right)$ and all of its closed subspaces are also reproducing kernel Hilbert spaces.

Proposition 2.1 Let $p \in \mathbb{C}[z]$ have degree at most $n$, let $\tilde{p}(z)=z^{n} \overline{p(1 / \bar{z})}$, and let

$$
d \mu=\frac{1}{|p(z)|^{2}} d \sigma(z)
$$

Then, with $B$ as in (2.3) the reproducing kernel for $L_{\mu}^{2}(B)$ is

$$
\mathcal{P}(z, \zeta)=(p(z) \overline{p(\zeta)}-\tilde{p}(z) \overline{\tilde{p}(\zeta)}) \mathcal{S}_{d}(z, \zeta)
$$

Proof The kernel for $L_{\mu}^{2}\left(\mathbb{Z}_{+}^{d}\right)$ is $p(z) \overline{p(\zeta)} \mathcal{S}_{d}(z, \zeta)$. This is a simple computation; if $f \in H^{2}\left(\mathbb{T}^{d}\right)$ and $\zeta \in \mathbb{D}^{d}$ then

$$
\begin{aligned}
\int_{\mathbb{T}^{d}} f(z) \overline{p(z) \overline{p(\zeta)} \mathcal{S}_{d}(z, \zeta)} d \mu(z) & =\int_{\mathbb{T}^{d}} f(z) \overline{p(z)} p(\zeta) \overline{\mathcal{S}_{d}(z, \zeta)} \frac{d \sigma(z)}{|p(z)|^{2}} \\
& =\int_{\mathbb{T}^{d}} \frac{f(z)}{p(z)} p(\zeta) \overline{\mathcal{S}_{d}(z, \zeta)} d \sigma(z) \\
& =\frac{f(\zeta)}{p(\zeta)} p(\zeta)=f(\zeta)
\end{aligned}
$$

The third equality is the reproducing property of $\mathcal{S}_{d}$ (or just the Cauchy integral formula).

We prove in Lemma 2.2 below that $L_{\mu}^{2}\left(\mathbb{Z}_{+}^{d} \ominus B\right)=\tilde{p} L_{\mu}^{2}\left(\mathbb{Z}_{+}^{d}\right)$ and a computation similar to that above proves that the reproducing kernel of $\tilde{p} L_{\mu}^{2}\left(\mathbb{Z}_{+}^{d}\right)$ is $\tilde{p}(z) \overline{\tilde{p}(\zeta)}$ $\mathcal{S}_{d}(z, \zeta)$. The result then follows from the fact that:

$$
L_{\mu}^{2}\left(\mathbb{Z}_{+}^{d}\right)=L_{\mu}^{2}(B) \oplus L_{\mu}^{2}\left(\mathbb{Z}_{+}^{d} \ominus B\right)
$$

and that the reproducing kernel of a direct sum is the sum of the reproducing kernels of each direct summand. Namely,

$$
\underbrace{p(z) \overline{p(\zeta)} \mathcal{S}_{d}(z, \zeta)}_{\text {kernel for } L_{\mu}^{2}\left(\mathbb{Z}_{+}^{d}\right)}-\underbrace{\tilde{p}(z) \overline{\tilde{p}(\zeta)} \mathcal{S}_{d}(z, \zeta)}_{\text {kernel for } L_{\mu}^{2}\left(\mathbb{Z}_{+}^{d} \ominus B\right)}=\text { kernel for } L_{\mu}^{2}(B) .
$$

The following lemma was used above.

\section{Lemma 2.2}

$$
\tilde{p} L_{\mu}^{2}\left(\mathbb{Z}_{+}^{d}\right)=L_{\mu}^{2}\left(\mathbb{Z}_{+}^{d} \ominus B\right)=L_{\mu}^{2}\left(\mathbb{Z}^{d} \ominus\left(\mathbb{Z}^{d} \backslash \mathbb{Z}_{n+}^{d}\right)\right)
$$


Proof Observe that $\tilde{p}(z)=z^{n} \overline{p(z)}$ on $\mathbb{T}^{d}$ and so

$$
\begin{aligned}
\left\langle z^{\alpha}, \tilde{p}\right\rangle_{\mu} & =\int_{\mathbb{T}^{d}} z^{\alpha} \overline{\tilde{p}(z)} \frac{1}{|p(z)|^{2}} d \sigma(z) \\
& =\int_{\mathbb{T}^{d}} z^{\alpha} \frac{\bar{z}^{n} p(z)}{|p(z)|^{2}} d \sigma(z) \\
& =\int_{\mathbb{T}^{d}} \frac{z^{\alpha-n}}{\overline{p(z)}} d \sigma(z) .
\end{aligned}
$$

This equals zero if any component of $\alpha-n$ is negative (i.e., if $\alpha \nsupseteq n$ ) since $1 / \bar{p}$ is anti-analytic in $\mathbb{D}^{d}$. In particular, if $\alpha \nsupseteq n$, then for $\beta \geq 0, \alpha \nsupseteq n+\beta$ and therefore

$$
\left\langle z^{\alpha}, z^{\beta} \tilde{p}\right\rangle_{\mu}=0
$$

This shows

$$
\tilde{p} L_{\mu}^{2}\left(\mathbb{Z}_{+}^{d}\right) \perp L_{\mu}^{2}\left(\mathbb{Z}^{d} \backslash \mathbb{Z}_{n+}^{d}\right)
$$

which means

$$
\tilde{p} L_{\mu}^{2}\left(\mathbb{Z}_{+}^{d}\right) \subset L_{\mu}^{2}\left(\mathbb{Z}^{d} \ominus\left(\mathbb{Z}^{d} \backslash \mathbb{Z}_{n+}^{d}\right)\right) \cap L_{\mu}^{2}\left(\mathbb{Z}_{+}^{d} \ominus B\right)
$$

Conversely, if $f \in L_{\mu}^{2}\left(\mathbb{Z}_{+}^{d} \ominus B\right)$ and $f \perp \tilde{p} L_{\mu}^{2}\left(\mathbb{Z}_{+}^{d}\right)$, then we can show $f \perp L_{\mu}^{2}\left(\mathbb{Z}_{+}^{d}\right)$ as follows.

Since $p(0) \neq 0, \tilde{p}(z)=a z^{n}+q(z)$ with $a=\overline{p(0)} \neq 0$ and $q$ of degree at most $n$ with no $z^{n}$ term. By assumption on $f, f \perp \tilde{p}$ and $f \perp q\left(q \in L_{\mu}^{2}(B)\right)$. Therefore, $f \perp z^{n}$. From here we can give an inductive proof on the lattice $\mathbb{Z}_{+}^{d}$. If $f$ is orthogonal to all non-negative frequencies less than some $\alpha \geq n$, then $f$ is orthogonal to

$$
z^{\alpha-n} \tilde{p}(z)=a z^{\alpha}+z^{\alpha-n} q(z) \quad \text { and } \quad z^{\alpha-n} q(z)
$$

as the latter contains only frequencies less than $\alpha$. This implies $f \perp z^{\alpha}$, and by induction $f \perp L_{\mu}^{2}\left(\mathbb{Z}_{+}^{d}\right)$. (As this is a non-traditional way of doing induction we should explain using the contrapositive: if $f$ is not perpendicular to some $z^{\alpha}$, then $f$ must also not be perpendicular to some $z^{\beta}$ with $\beta<\alpha$. This can be continued until $f$ is not perpendicular to a monomial supported in $B-$ a contradiction.) This forces $f \equiv 0$.

Hence, $L_{\mu}^{2}\left(\mathbb{Z}_{+}^{d} \ominus B\right)=\tilde{p} L_{\mu}^{2}\left(\mathbb{Z}_{+}^{d}\right) \subset L_{\mu}^{2}\left(\mathbb{Z}^{d} \ominus\left(\mathbb{Z}^{d} \backslash \mathbb{Z}_{n+}^{d}\right)\right)$. By Lemma 2.3 given below, we automatically have

$$
L_{\mu}^{2}\left(\mathbb{Z}_{+}^{d} \ominus B\right)=\tilde{p} L_{\mu}^{2}\left(\mathbb{Z}_{+}^{d}\right)=L_{\mu}^{2}\left(\mathbb{Z}^{d} \ominus\left(\mathbb{Z}^{d} \backslash \mathbb{Z}_{n+}^{d}\right)\right)
$$


Lemma 2.3 Suppose $W, Y \subset \mathbb{Z}^{d}$ and set $X=W \cup Y$. Then,

$$
L_{\mu}^{2}(X) \ominus L_{\mu}^{2}(Y) \subset L_{\mu}^{2}(W)
$$

if and only if

$$
L_{\mu}^{2}(W) \ominus L_{\mu}^{2}(Y \cap W) \subset\left(L_{\mu}^{2}(Y)\right)^{\perp}
$$

and in either case

$$
L_{\mu}^{2}(X) \ominus L_{\mu}^{2}(Y)=L_{\mu}^{2}(W) \ominus L_{\mu}^{2}(Y \cap W) .
$$

Proof This is essentially a result of the decomposition

$$
\begin{aligned}
L_{\mu}^{2}(X \ominus(Y \cap W)) & =L_{\mu}^{2}(X \ominus Y) \oplus L_{\mu}^{2}(Y \ominus(Y \cap W)) \\
& =L_{\mu}^{2}(X \ominus W) \oplus L_{\mu}^{2}(W \ominus(Y \cap W)) .
\end{aligned}
$$

Suppose $L_{\mu}^{2}(X \ominus Y) \subset L_{\mu}^{2}(W)$ which necessarily means $L_{\mu}^{2}(X \ominus Y) \subset L_{\mu}^{2}(W \ominus$ $(Y \cap W))$. If $f \in L_{\mu}^{2}(W \ominus(Y \cap W)) \ominus L_{\mu}^{2}(X \ominus Y)$, then $f \in L_{\mu}^{2}(Y \ominus(Y \cap W))$ by (2.6). Hence, $f \in L_{\mu}^{2}(Y \cap W \ominus Y \cap W)=\{0\}$ showing that $L_{\mu}^{2}(X \ominus Y)$ fills out all of $L_{\mu}^{2}(W \ominus(Y \cap W))$.

Suppose $L_{\mu}^{2}(W \ominus(Y \cap W)) \subset L_{\mu}^{2}(Y)^{\perp}$ which necessarily means $L_{\mu}^{2}(W \ominus(Y \cap$ $W)) \subset L_{\mu}^{2}(X \ominus Y)$. If $f \in L_{\mu}^{2}(X \ominus Y) \ominus L_{\mu}^{2}(W \ominus(Y \cap W))$, then $f \in L_{\mu}^{2}(X \ominus W)$ by (2.7). Hence, $f \perp L_{\mu}^{2}(Y)+L_{\mu}^{2}(W)=L_{\mu}^{2}(X)$, forcing $f \equiv 0$. This shows that $L_{\mu}^{2}(W \ominus(Y \cap W))$ fills out all of $L_{\mu}^{2}(X \ominus Y)$.

So, we have shown that $\mathcal{P}$ represents the reproducing kernel of $L_{\mu}^{2}(B)$. Any orthogonal decomposition of $L_{\mu}^{2}(B)$ then gives a decomposition of $\mathcal{P}$. Our goal is to prove that $L_{\mu}^{2}(B)$ has a decomposition with very special properties.

\section{Orthogonal Decompositions of $L_{\mu}^{2}(B)$}

We recall the definition of $B$ and define several subsets of $B$ below:

\section{Notation 3.1}

$$
\begin{aligned}
X_{j} & :=\left\{\alpha \in \mathbb{Z}_{+}^{d}: \alpha_{j}<n_{j}\right\} \\
X_{S} & :=\bigcup_{j \in S} X_{j}=\left\{\alpha \in \mathbb{Z}_{+}^{d}: \exists j \in S: \alpha_{j}<n_{j}\right\} \\
B & =\bigcup_{j=1}^{d} X_{j}=\left\{\alpha \in \mathbb{Z}_{+}^{d}: \exists j: \alpha_{j}<n_{j}\right\}
\end{aligned}
$$

where $S \subset\{1,2, \ldots, d\}$. 
Proposition 3.2 With the same setup as Proposition 2.1 let $S \sqcup T=\{1,2, \ldots, d\}$ be a partition. Then,

$$
L_{\mu}^{2}(B)=L_{\mu}^{2}\left(X_{T}\right) \oplus L_{\mu}^{2}\left(X_{S} \ominus\left(X_{S} \cap X_{T}\right)\right) .
$$

The content of the above proposition is that the subspaces listed in the orthogonal decomposition are actually orthogonal, something which would not hold for a general finite measure on $\mathbb{T}^{d}$. This proposition is still valid if $S$ or $T$ are empty if we interpret $X_{\varnothing}=\{0\}$. This makes the proposition sensible (although trivial) in the case $d=1$ (something useful later).

We need the following notation for use in dividing up all of structures according to the partition $S \sqcup T=\{1, \ldots, d\}$. There is no harm in assuming $S=\{1, \ldots, s\}$, $T=\{s+1, \ldots, d\}$, and $t:=d-s$.

$$
\begin{aligned}
z_{S}=\left(z_{1}, \ldots, z_{S}\right) \in \mathbb{C}^{S}, & z_{T}=\left(z_{s+1}, \ldots, z_{d}\right) \in \mathbb{C}^{t}, & z=\left(z_{S}, z_{T}\right) \\
n_{S}=\left(n_{1}, \ldots, n_{S}\right) \in \mathbb{Z}^{s}, & n_{T}=\left(n_{s+1}, \ldots, n_{d}\right) \in \mathbb{Z}^{t}, & n=\left(n_{S}, n_{T}\right) \\
\alpha_{S}=\left(\alpha_{1}, \ldots, \alpha_{S}\right) \in \mathbb{Z}^{s}, & \alpha_{T}=\left(\alpha_{S+1}, \ldots, \alpha_{d}\right) \in \mathbb{Z}^{t}, & \alpha=\left(\alpha_{S}, \alpha_{T}\right) \\
B_{S}=\left\{\alpha_{S} \in \mathbb{Z}_{+}^{S}: \alpha_{S} \ngtr n_{S}\right\}, & B_{T}=\left\{\alpha_{T} \in \mathbb{Z}_{+}^{t}: \alpha_{T} \ngtr n_{T}\right\} &
\end{aligned}
$$

It is useful to note here that

$$
\begin{array}{lr}
X_{S}=B_{S} \times \mathbb{Z}_{+}^{t}, & X_{T}=\mathbb{Z}_{+}^{S} \times B_{T}, \\
B_{S} \times B_{T}=X_{S} \cap X_{T}, & B=X_{S} \cup X_{T}
\end{array}
$$

Proof of Proposition 3.2 The proposition is really a type of inclusion-exclusion principle as it can be rewritten as saying

$$
L_{\mu}^{2}\left(\left(X_{S} \cup X_{T}\right) \ominus X_{T}\right)=L_{\mu}^{2}\left(X_{S} \ominus\left(X_{S} \cap X_{T}\right)\right) .
$$

To prove it, consider following the measures $\mu_{z_{S}}$ on $\mathbb{T}^{t}$ which are indexed by $z_{S} \in \mathbb{T}^{s}:$

$$
d \mu_{z_{S}}\left(z_{T}\right)=\frac{1}{\left|p\left(z_{S}, z_{T}\right)\right|^{2}} d \sigma\left(z_{T}\right)
$$

i.e. for each $z_{S} \in \mathbb{T}^{s}$ we get a measure on $\mathbb{T}^{t}$, and points in $\mathbb{T}^{t}$ are denoted by $z_{T}$.

By Proposition 2.1, the reproducing kernel for $L_{\mu_{z S}}^{2}\left(B_{T}\right)$ is

$$
\mathcal{P}_{z_{S}}^{T}\left(z_{T}, \zeta_{T}\right):=\left(p\left(z_{S}, z_{T}\right) \overline{p\left(z_{S}, \zeta_{T}\right)}-\tilde{p}\left(z_{S}, z_{T}\right) \overline{\tilde{p}\left(z_{S}, \zeta_{T}\right)}\right) \mathcal{S}_{t}\left(z_{T}, \zeta_{T}\right)
$$

where again $\mathcal{S}_{t}$ is the $t$-dimensional Szegó kernel. Notice that $\mathcal{P}_{z_{S}}^{T}\left(z_{T}, \zeta_{T}\right)$ is a trigonometric polynomial of degree at most $n_{S}$ as a function of $z_{S}$, while as a function of $z_{T}$ this function only has Fourier coefficients corresponding to points of $B_{T}$. For these reasons, the function of $z=\left(z_{S}, z_{T}\right) \in \mathbb{T}^{d}$ defined for each fixed $\zeta \in \mathbb{D}^{d}$ by

$$
L_{\zeta}(z)=L(z, \zeta)=z_{S}^{n_{S}} \bar{\zeta}_{S}^{n_{S}} \mathcal{S}_{S}\left(z_{S}, \zeta_{S}\right) \mathcal{P}_{z_{S}}^{T}\left(z_{T}, \zeta_{T}\right)
$$


is in $L_{\mu}^{2}\left(\mathbb{Z}_{+}^{S} \times B_{T}\right)=L_{\mu}^{2}\left(X_{T}\right)$. (Specifically, as a function of $\left(z_{S}, z_{T}\right)$

$$
\begin{aligned}
\mathcal{S}_{S}\left(z_{S}, \zeta_{S}\right) & \in L_{\mu}^{2}\left(\mathbb{Z}_{+}^{S} \times\left\{0_{T}\right\}\right) \\
\mathcal{P}_{z_{S}}^{T}\left(z_{T}, \zeta_{T}\right) & \in L_{\mu}^{2}\left(\left[-n_{S}, n_{S}\right] \times B_{T}\right)
\end{aligned}
$$

Here $0_{T}$ is the zero $t$-tuple in $\mathbb{Z}^{t}$ and $\left[-n_{S}, n_{S}\right]=\left\{\alpha_{S} \in \mathbb{Z}^{S}:-n_{S} \leq \alpha_{S} \leq n_{S}\right\}$.) So, if $f \perp L_{\mu}^{2}\left(X_{T}\right)$, then

$$
\left\langle f, L_{\zeta}\right\rangle_{\mu}=0 \text { for all } \zeta \in \mathbb{D}^{d} \text {. }
$$

On the other hand, $L$ can be thought of as a difference of two terms:

$$
\begin{aligned}
L_{\zeta}(z)= & \underbrace{p\left(z_{S}, z_{T}\right) \overline{p\left(z_{S}, \zeta_{T}\right)}\left(z_{S}^{n_{S}} \bar{\zeta}_{S}^{n_{S}}\right) \mathcal{S}_{d}(z, \zeta)}_{A_{\zeta}} \\
& \underbrace{-\tilde{p}\left(z_{S}, z_{T}\right) \overline{\tilde{p}\left(z_{S}, \zeta_{T}\right)}\left(z_{S}^{n_{S}} \bar{\zeta}_{S}^{n_{S}}\right) \mathcal{S}_{d}(z, \zeta)}_{B_{\zeta}} .
\end{aligned}
$$

(We used $\mathcal{S}_{d}(z, \zeta)=\mathcal{S}_{S}\left(z_{S}, \zeta_{S}\right) \mathcal{S}_{t}\left(z_{T}, \zeta_{T}\right)$ above.)

Since $z_{S}^{n_{S}} \overline{\tilde{p}\left(z_{S}, \zeta_{T}\right)}$ has only non-negative Fourier coefficients in $z_{S}$, the second term $B_{\zeta}$ is an element of $\tilde{p} L_{\mu}^{2}\left(\mathbb{Z}_{+}^{d}\right)=L_{\mu}^{2}\left(\mathbb{Z}_{+}^{d} \ominus B\right)$. So, if $f \in L_{\mu}^{2}(B)$, then $B_{\zeta} \perp f$ and we have

$$
\left\langle f, L_{\zeta}\right\rangle_{\mu}=\left\langle f, A_{\zeta}\right\rangle_{\mu}
$$

Finally, if $f \in L_{\mu}^{2}\left(\mathbb{Z}_{+}^{d}\right)$ then

$$
\begin{aligned}
\left\langle f, A_{\zeta}\right\rangle_{\mu} & =\int_{\mathbb{T}^{s}} \int_{\mathbb{T}^{t}} f(z) \overline{p(z)} p\left(z_{S}, \zeta_{T}\right) \overline{\mathcal{S}_{t}\left(z_{T}, \zeta_{T}\right)} \frac{d \sigma\left(z_{T}\right)}{|p(z)|^{2}}\left(\overline{z_{S}}{ }^{n_{S}} \zeta_{S}^{n_{S}}\right) \overline{\mathcal{S}_{S}\left(z_{S}, \zeta_{S}\right)} d \sigma\left(z_{S}\right) \\
& =\int_{\mathbb{T}^{s}} \int_{\mathbb{T}^{t}} \frac{f(z)}{p(z)} p\left(z_{S}, \zeta_{T}\right) \overline{\mathcal{S}_{t}\left(z_{T}, \zeta_{T}\right)} d \sigma\left(z_{T}\right)\left(\overline{z_{S}} n_{S} \zeta_{S}^{n_{S}}\right) \overline{\mathcal{S}_{S}\left(z_{S}, \zeta_{S}\right)} d \sigma\left(z_{S}\right) \\
& =\int_{\mathbb{T}^{s}} \frac{f\left(z_{S}, \zeta_{T}\right)}{p\left(z_{S}, \zeta_{T}\right)} p\left(z_{S}, \zeta_{T}\right)\left(\overline{z_{S} n_{S}} \zeta_{S}^{n_{S}}\right) \overline{\mathcal{S}_{S}\left(z_{S}, \zeta_{S}\right)} d \sigma\left(z_{S}\right) \\
& =\int_{\mathbb{T}^{s}} f\left(z_{S}, \zeta_{T}\right)\left(\overline{z_{S}}{ }^{n_{S}} \zeta_{S}^{n_{S}}\right) \overline{\mathcal{S}_{S}\left(z_{S}, \zeta_{S}\right)} d \sigma\left(z_{S}\right) \\
& =\sum_{\alpha_{S} \geq n_{S}} \sum_{\alpha_{T} \geq 0} \hat{f\left(\alpha_{S}, \alpha_{T}\right) \zeta^{\alpha}}
\end{aligned}
$$

which is the $L^{2}\left(\mathbb{T}^{d}\right)$ projection of $f$ to $z_{S}^{n_{S}} H^{2}\left(\mathbb{T}^{d}\right)$. (The second and fourth equalities are algebra, the third is the reproducing property of $\mathcal{S}_{t}$, and the fifth is a Fourier series computation.) 
If we combine the observations (3.1)-(3.3) above we see that if

$$
f \perp L_{\mu}^{2}\left(X_{T}\right) \quad \text { and } \quad f \in L_{\mu}^{2}(B)
$$

then

$$
\hat{f}(\alpha)=0
$$

for $\alpha_{S} \geq n_{S}, \alpha_{T} \geq 0$ and therefore $f \in L_{\mu}^{2}\left(X_{S}\right)$. So, $L_{\mu}^{2}\left(B \ominus X_{T}\right) \subset L_{\mu}^{2}\left(X_{S}\right)$.

By Lemma 2.3, this proves

$$
L_{\mu}^{2}\left(B \ominus X_{T}\right)=L_{\mu}^{2}\left(X_{S} \ominus\left(X_{S} \cap X_{T}\right)\right)
$$

since $B=X_{S} \cup X_{T}$.

\section{Closed Under Shifts}

The goal of this section is to prove two facts.

Proposition 4.1 With the setup of Proposition 3.2, $L_{\mu}^{2}\left(X_{S}\right)$ is closed under multiplication by $z_{j}$ for all $j \notin S$, and contains all subspaces of $L_{\mu}^{2}(B)$ with this property.

Proposition 4.2 With the setup of Proposition 3.2, $L_{\mu}^{2}\left(X_{S} \ominus\left(X_{S} \cap X_{T}\right)\right)$ is closed under multiplication by $z_{j}$ for all $j \in T$.

The first fact is not difficult.

Proof of Proposition 4.1 An element $f \in L_{\mu}^{2}(B)$ is in $L_{\mu}^{2}\left(X_{S}\right)$ if and only if $\hat{f}(\alpha)=0$ whenever $\alpha_{k} \geq n_{k}$ for all $k \in S$. This property is obviously unaffected by multiplying $f$ by variables $z_{j}$ for $j \notin S$.

On the other hand, if $f \in L_{\mu}^{2}(B)$, has the property that

$$
z^{\alpha} f \in L_{\mu}^{2}(B)
$$

for all $\alpha \geq 0$ satisfying $\alpha_{j}=0$ for $j \in S$, then $f$ must be an element of $L_{\mu}^{2}\left(X_{S}\right)$. Otherwise, $\hat{f}(\alpha) \neq 0$ for some $\alpha \geq 0$, with $\alpha_{k} \geq n_{k}$ for all $k \in S$. But then if we set $m=\left(m_{1}, \ldots, m_{d}\right)$ where

$$
m_{j}= \begin{cases}0 & \text { for } j \in S \\ n_{j} & \text { for } j \notin S\end{cases}
$$

then $z^{m} f \notin L_{\mu}^{2}(B)$ - a contradiction. This proves that $L_{\mu}^{2}\left(X_{S}\right)$ contains all subspaces closed under multiplication by all $z_{j}$ for $j \notin S$. 
As for Proposition 4.2, it is convenient to prove the proposition by adjoining a variable and using results in $d$ variables that have already been proven. Elements of $\mathbb{C}^{d+1}=\mathbb{C} \times \mathbb{C}^{d}$ will be written as $\left(z_{0}, z\right)$. So, now $p \in \mathbb{C}\left[z_{0}, z\right]$ is a polynomial of $d+1$ variables of degree at most $\left(n_{0}, n\right)$ with no zeros in $\overline{\mathbb{D}^{d+1}}$. The measure $\mu$ corresponds to $\left|p\left(z_{0}, z\right)\right|^{-2} d \sigma\left(z_{0}, z\right)$.

Notation already defined for $d$ variables will retain its meaning, while we will use the following notation for certain $d+1$-variable objects:

$$
\begin{aligned}
& Y_{j}=\left\{\left(\alpha_{0}, \alpha\right) \in \mathbb{Z}_{+}^{d+1}: \alpha_{j}<n_{j}\right\} \\
& Y_{S}=\bigcup_{j \in S} Y_{j} \text { for } S \subset\{0,1, \ldots, d\}
\end{aligned}
$$

We also find it convenient to use interval notation for subsets of integers (as opposed to real numbers):

$$
\begin{aligned}
& (a, b)=\{k \in \mathbb{Z}: a<k<b\} \\
& {[a, b)=\{k \in \mathbb{Z}: a \leq k<b\}, \text { etc. }}
\end{aligned}
$$

We never make use of intervals of real numbers, so there should be no confusion.

Now, let $S \sqcup T$ be a partition of $\{1, \ldots, d\}$, and let $T_{0}=T \cup\{0\}$. We will prove that

$$
L_{\mu}^{2}\left(Y_{S} \ominus\left(Y_{T_{0}} \cap Y_{S}\right)\right)
$$

is closed under multiplication by $z_{0}$. This is enough to prove the proposition.

Proof of Proposition 4.2 For each $z_{0} \in \mathbb{T}$, let $d \mu_{z_{0}}(z)$ be the measure on $\mathbb{T}^{d}$

$$
d \mu_{z_{0}}(z)=\frac{1}{\left|p\left(z_{0}, z\right)\right|^{2}} d \sigma(z) .
$$

Let

$$
\Gamma_{z_{0}}(z, \zeta)
$$

denote the reproducing kernel for $L_{\mu_{z_{0}}}^{2}\left(X_{T} \ominus\left(X_{T} \cap X_{S}\right)\right)$, and let

$$
\Delta_{z_{0}}(z, \zeta)
$$

denote the reproducing kernel for $L_{\mu_{z_{0}}}^{2}\left(X_{S}\right)$.

By Proposition 3.2,

$$
\begin{aligned}
& \left(p\left(z_{0}, z\right) \overline{p\left(z_{0}, \zeta\right)}-\tilde{p}\left(z_{0}, z\right) \overline{\tilde{p}\left(z_{0}, \zeta\right)}\right) \mathcal{S}_{d}(z, \zeta) \\
& \quad=\Gamma_{z_{0}}(z, \zeta)+\Delta_{z_{0}}(z, \zeta)
\end{aligned}
$$


The left hand side is a trigonometric polynomial in $z_{0}$ of degree at most $n_{0}$, while $\Delta_{z_{0}}(z, \zeta)$ as a function of $z$ is the only function on the right hand side with any Fourier support in $X_{S} \backslash X_{T}$. This means the coefficients of $z^{\alpha}$ in $\Delta_{z_{0}}$ for $\alpha \in X_{S} \backslash X_{T}$ are trigonometric polynomials with respect to $z_{0}$; i.e. as a function of $\left(z_{0}, z\right)$

$$
\begin{aligned}
& \Delta_{z_{0}}(z, \zeta) \in L_{\mu}^{2}\left(\mathbb{Z} \times\left(X_{S} \cap X_{T}\right) \cup\left[-n_{0}, n_{0}\right] \times\left(X_{S} \backslash X_{T}\right)\right) \\
& =L_{\mu}^{2}\left(\mathbb{Z} \times\left(X_{S} \cap X_{T}\right) \cup\left[-n_{0}, n_{0}\right] \times X_{S}\right)
\end{aligned}
$$

(Perhaps it needs to be explicitly stated that $\Delta_{z_{0}}(z, \zeta)$ is actually in $L^{2}\left(\mathbb{T}^{d+1}\right)$ as a function of $\left(z_{0}, z\right) \in \mathbb{T}^{d+1}$. See Lemma 4.3 below.)

Define for each $Z=\left(\zeta_{0}, \zeta\right) \in \mathbb{D}^{d+1}$

$$
L_{Z}\left(z_{0}, z\right)=L\left(\left(z_{0}, z\right), Z\right)=\frac{\overline{z_{0}} \zeta_{0}}{1-\overline{z_{0}} \zeta_{0}} \Delta_{z_{0}}(z, \zeta)
$$

By (4.1) and (4.2),

$$
L_{Z} \in L_{\mu}^{2}\left(\mathbb{Z} \times\left(X_{S} \cap X_{T}\right) \cup\left(-\infty, n_{0}\right) \times X_{S}\right) .
$$

Now, let $f \in L_{\mu}^{2}\left(\mathbb{Z} \times X_{S}\right)$, then for each $Z=\left(\zeta_{0}, \zeta\right) \in \mathbb{D}^{d+1}$

$$
\begin{aligned}
\left\langle f, L_{Z}\right\rangle_{\mu} & =\int_{\mathbb{T}_{\mathbb{T}^{d}}} f\left(z_{0}, z\right) \overline{\Delta_{z_{0}}(z, \zeta)} d \mu_{z_{0}}(z) \frac{z_{0} \bar{\zeta}_{0}}{1-z_{0} \overline{\zeta_{0}}} d \sigma\left(z_{0}\right) \\
& =\int_{\mathbb{T}} f\left(z_{0}, \zeta\right) \frac{z_{0} \bar{\zeta}_{0}}{1-z_{0} \bar{\zeta}_{0}} d \sigma\left(z_{0}\right) \\
& =\sum_{\alpha_{0}=-\infty} \sum_{\alpha \geq 0} \hat{f}\left(\alpha_{0}, \alpha\right) \bar{\zeta}_{0}^{-\alpha_{0}} \zeta^{\alpha} ;
\end{aligned}
$$

the equality (4.3) is by definition, (4.4) is because $\Delta_{z_{0}}$ is a reproducing kernel for $X_{S}$ with respect to $\mu_{z_{0}}$, and (4.5) is a Fourier series computation. If

$$
\begin{aligned}
& f \in L_{\mu}^{2}\left(\mathbb{Z} \times X_{S}\right) \quad \text { and } \\
& f \perp L_{\mu}^{2}\left(\mathbb{Z} \times\left(X_{S} \cap X_{T}\right) \cup\left(-\infty, n_{0}\right) \times X_{S}\right)
\end{aligned}
$$

then $f \perp L_{Z}$ and therefore the expression in (4.5) is zero which implies $f \in L_{\mu}^{2}$ $\left(\mathbb{Z}_{+} \times X_{S}\right)=L_{\mu}^{2}\left(Y_{S}\right)$. Hence, by Lemma 2.3

$$
L_{\mu}^{2}\left(\mathbb{Z} \times X_{S}\right) \ominus L_{\mu}^{2}\left(\mathbb{Z} \times\left(X_{S} \cap X_{T}\right) \cup\left(-\infty, n_{0}\right) \times X_{S}\right)
$$

is unchanged if we intersect all sets with $Y_{S}$. This proves (4.6) equals

$$
L_{\mu}^{2}\left(Y_{S}\right) \ominus L_{\mu}^{2}\left(Y_{S} \cap Y_{T_{0}}\right)
$$


where we are using the facts that

$$
\left(\mathbb{Z} \times X_{S}\right) \cap Y_{S}=Y_{S}
$$

and

$$
\begin{aligned}
(\mathbb{Z} & \left.\times\left(X_{S} \cap X_{T}\right) \cup\left(-\infty, n_{0}\right) \times X_{S}\right) \cap Y_{S} \\
& =\left(Y_{S} \cap Y_{T}\right) \cup\left(Y_{S} \cap Y_{\{0\}}\right) \\
& =Y_{S} \cap Y_{T_{0}} .
\end{aligned}
$$

This proves

$$
L_{\mu}^{2}\left(Y_{S} \ominus\left(Y_{S} \cap Y_{T_{0}}\right)\right) \perp L_{\mu}^{2}\left((-\infty, 0) \times X_{S}\right)
$$

since $(4.6)=(4.7)$ and since

$$
(-\infty, 0) \times X_{S} \subset \mathbb{Z} \times\left(X_{S} \cap X_{T}\right) \cup\left(-\infty, n_{0}\right) \times\left(X_{S} \backslash X_{T}\right) .
$$

This is enough to show $L_{\mu}^{2}\left(Y_{S} \ominus\left(Y_{S} \cap Y_{T_{0}}\right)\right)$ is closed under multiplication by $z_{0}$, as follows.

Let $f \in L_{\mu}^{2}\left(Y_{S} \ominus\left(Y_{S} \cap Y_{T_{0}}\right)\right)$. By Proposition 4.1, it is clear that $z_{0} f \in L_{\mu}^{2}\left(Y_{S}\right)$. To show $z_{0} f \perp L_{\mu}^{2}\left(Y_{S} \cap Y_{T_{0}}\right)$, let $\left(\alpha_{0}, \alpha\right) \in Y_{S} \cap Y_{T_{0}}$. If $\alpha_{0}>0$ then $\left(\alpha_{0}-1, \alpha\right) \in Y_{S} \cap Y_{T_{0}}$ in which case

$$
\left\langle z_{0} f, z_{0}^{\alpha_{0}} z^{\alpha}\right\rangle_{\mu}=\left\langle f, z_{0}^{\alpha_{0}-1} z^{\alpha}\right\rangle_{\mu}=0
$$

If $\alpha_{0}=0$, then $\left(\alpha_{0}-1, \alpha\right) \in(-\infty, 0) \times X_{S}$ in which case we again have (4.8) because $f \perp L_{\mu}^{2}\left((-\infty, 0) \times X_{S}\right)$. Hence, $z_{0} f \in L_{\mu}^{2}\left(Y_{S} \ominus\left(Y_{S} \cap Y_{T_{0}}\right)\right)$, proving that this subspace is closed under multiplication by $z_{0}$.

We used the following lemma in the above proof.

Lemma 4.3 Let $X \subset \mathbb{Z}_{+}^{d}, \zeta \in \mathbb{D}^{d}$. The reproducing kernel of $L_{\mu_{z_{0}}}^{2}(X)$, written $K_{z_{0}}(X)(z, \zeta)$ is in $L^{2}\left(\mathbb{T}^{d+1}\right)$ as a function of $\left(z_{0}, z\right)$.

Proof For each $\alpha \in \mathbb{Z}^{d}$, let

$$
C_{\alpha}\left(z_{0}\right)=\int_{\mathbb{T}^{d}} \frac{z^{\alpha}}{\left|p\left(z_{0}, z\right)\right|^{2}} d \sigma(z)
$$

and define the following (generally infinite) self-adjoint matrix indexed by $X$

$$
C_{X}\left(z_{0}\right)=\left(C_{\alpha-\beta}\left(z_{0}\right)\right)_{\alpha, \beta \in X}
$$


The entries of $C_{X}\left(z_{0}\right)$ are clearly continuous on $\mathbb{T}$. Since $|p|$ is bounded above and below on the circle, it turns out $C_{X}\left(z_{0}\right)$ is bounded above and below as an operator on $\ell^{2}(X)$. Indeed, for $\left(v_{\alpha}\right) \in \ell^{2}(X)$

$$
\sum_{\alpha, \beta \in X} C_{\alpha-\beta}\left(z_{0}\right) v_{\alpha} \bar{v}_{\beta}=\int_{\mathbb{T}^{d}} \frac{\left|\sum_{\alpha \in X} v_{\alpha} z^{\alpha}\right|^{2}}{\left|p\left(z_{0}, z\right)\right|^{2}} d \sigma(z)
$$

is bounded above and below by

$$
\int_{\mathbb{T}^{d}}\left|\sum_{\alpha \in X} v_{\alpha} z^{\alpha}\right|^{2} d \sigma(z)=\sum_{\alpha \in X}\left|v_{\alpha}\right|^{2}
$$

with constants $c_{1}=\left(\inf _{\mathbb{T}^{d+1}}|p|\right)^{-2}$ and $c_{2}=\left(\sup _{\mathbb{T}^{d+1}}|p|\right)^{-2}$, respectively.

Let

$$
B_{\alpha, \beta}\left(z_{0}\right)=\left(C_{X}\left(z_{0}\right)\right)_{\alpha, \beta}^{-1}
$$

be the $(\alpha, \beta)$ entry of the inverse of $C_{X}\left(z_{0}\right)$. The reproducing kernel $K_{z_{0}}(X)(z, \zeta)$ can be given explicitly as

$$
K_{z_{0}}(X)(z, \zeta)=\sum_{\alpha, \beta \in X} B_{\beta, \alpha}\left(z_{0}\right) z^{\alpha}(\bar{\zeta})^{\beta}
$$

The proof of this fact is a direct computation; if $\gamma \in X$, then

$$
\left\langle z^{\gamma} \sum_{\alpha, \beta \in X} B_{\beta, \alpha}\left(z_{0}\right) z^{\alpha}(\bar{\zeta})^{\beta}\right\rangle_{\mu_{z_{0}}}=\sum_{\alpha, \beta \in X} C_{\gamma-\alpha}\left(z_{0}\right) B_{\alpha, \beta}\left(z_{0}\right) \zeta^{\beta}=\zeta^{\gamma}
$$

Since $C_{X}\left(z_{0}\right)$ is bounded above and below,

$$
\sum_{\alpha \in X}\left(\sum_{\beta \in X} B_{\alpha, \beta}\left(z_{0}\right)(\bar{\zeta})^{\beta}\right) z^{\alpha}
$$

is in $L^{2}\left(\mathbb{T}^{d+1}\right)$ as a function of $\left(z_{0}, z\right)$ for each $\zeta \in \mathbb{D}^{d}$.

\section{Proof of Theorem 1.5}

So far we have shown (in Proposition 3.2)

$$
L_{\mu}^{2}(B)=L_{\mu}^{2}\left(X_{T}\right) \oplus L_{\mu}^{2}\left(X_{S} \ominus\left(X_{S} \cap X_{T}\right)\right)
$$


for each partition $S \sqcup T=\{1, \ldots, d\}$. In addition, $L_{\mu}^{2}\left(X_{S}\right)$ and $L_{\mu}^{2}\left(X_{S} \ominus\left(X_{S} \cap X_{T}\right)\right)$ are closed under multiplication by all variables $z_{j}$ for $j \in T$ and $L_{\mu}^{2}\left(X_{S}\right)$ is maximal among subspaces with this property (Propositions 4.1 and 4.2).

Theorem 1.5 now reduces to bookkeeping and facts about reproducing kernels. Namely, a kernel is a $\mathcal{P}$-kernel if it is the reproducing kernel for a closed subspace of $L_{\mu}^{2}(B)$ (Lemma 7.5). For a non-empty $S \subset\{1, \ldots, d\}$, set $T=\{1, \ldots, d\} \backslash S$ and let

- $K_{S}$ be the reproducing kernel for $L_{\mu}^{2}\left(X_{T}\right)$ and

- $L_{S}$ be the reproducing kernel for $L_{\mu}^{2}\left(X_{T} \ominus\left(X_{S} \cap X_{T}\right)\right)$

(these definitions look like $S$ and $T$ have been mistakenly switched but they have not). Both $K_{S}$ and $L_{S}$ are $S$-contractive $\mathcal{P}$-kernels by Lemma 7.7 and Propositions 4.1 and 4.2.

By Proposition 3.2 we have

$$
\mathcal{P}=K_{S}+L_{T}
$$

To prove the maximality property of $K_{S}$, suppose $\mathcal{P} \geq K \geq 0$ for some $S$-contractive kernel $K$. By Lemmas 7.2 and 7.6 below, $z^{\alpha} K_{\zeta} \in L_{\mu}^{2}(B)$ for all $\zeta \in \mathbb{D}^{d}$ and all $\alpha \geq 0$ satisfying $\alpha_{j}=0$ for $j \notin S$. By Proposition 4.1, $K_{\zeta} \in L_{\mu}^{2}\left(X_{T}\right)$ and therefore by Lemma $7.4, K_{S}$ must dominate $K$ :

$$
K_{S} \geq K
$$

This completes the proof of Theorem 1.5.

\section{Proof of Theorem 1.3}

We have already proven the theorem for rational inner functions which are regular on $\overline{\mathbb{D}^{d}}$, since such functions can always be represented by $f=\tilde{p} / p$ where $p \in \mathbb{C}[z]$ with no zeros on $\overline{\mathbb{D}^{d}}$. Namely, we have by Theorem 1.5

$$
\frac{1-f(z) \overline{f(\zeta)}}{\prod_{j=1}^{d}\left(1-z_{j} \bar{\zeta}_{j}\right)}=\frac{K_{S}(z, \zeta)}{p(z) \overline{p(\zeta)}}+\frac{L_{T}(z, z)}{p(z) \overline{p(\zeta)}}
$$

Let us agree to absorb the denominators into the definitions of $K_{S}$ and $L_{T}$ so that we really have the formula

$$
\frac{1-f(z) \overline{f(\zeta)}}{\prod_{j=1}^{d}\left(1-z_{j} \bar{\zeta}_{j}\right)}=K_{S}(z, \zeta)+L_{T}(z, \zeta)
$$

By Theorem 1.5, $K_{S}+L_{T}=K_{T}+L_{S}$ and by maximality of $K_{S}, K_{T}$ among $S$ and $T$-contractive $\mathcal{P}$-kernels, respectively, we have

$$
K_{S}-L_{S}=K_{T}-L_{T} \geq 0
$$


and

$$
K_{S} \geq K_{S^{\prime}} \text { for } S \subset S^{\prime}
$$

To prove the theorem for a general holomorphic function $f: \mathbb{D}^{d} \rightarrow \mathbb{D}$, we use a theorem of Rudin [5, Theorem 5.5.1] which says that such $f$ can be approximated uniformly on compact subsets of $\mathbb{D}^{d}$ by rational inner functions, regular on $\overline{\mathbb{D}^{d}}$. So, say $f_{k} \rightarrow f$ uniformly on compacta, with each $f_{k}$ rational, inner, and continuous up to $\overline{\mathbb{D}^{d}}$. We have corresponding decompositions:

$$
\frac{1-f_{k}(z) \overline{f_{k}(\zeta)}}{\prod_{j=1}^{d}\left(1-z_{j} \bar{\zeta}_{j}\right)}=K_{S}^{(k)}(z, \zeta)+L_{T}^{(k)}(z, \zeta)
$$

Since

$$
\left|K_{S}^{(k)}(z, \zeta)\right|^{2} \leq K_{S}^{(k)}(z, z) K_{S}^{(k)}(\zeta, \zeta) \leq \frac{1}{\prod_{j=1}^{d}\left(1-\left|z_{j}\right|^{2}\right)\left(1-\left|\zeta_{j}\right|^{2}\right)}
$$

(with $L_{T}^{(k)}$ satisfying a similar estimate), we see that the $K_{S}^{(k)}$, s and $L_{T}^{(k)}$, s are holomorphic on $\mathbb{D}^{d} \times \mathbb{D}^{d}$ and locally uniformly bounded and hence they are in a normal family. Taking subsequences, we may assume $K_{S}^{(k)}$ converges to some $K_{S}$ and $L_{T}^{(k)}$ converges to some $L_{T}$ locally uniformly. Positive semi-definiteness, $S$ and $T$ contractivity, and the identities/inequalities

$$
\begin{aligned}
& K_{S}-L_{S}=K_{T}-L_{T} \geq 0 \\
& K_{S} \geq K_{S}^{\prime} \text { for } S \subset S^{\prime}
\end{aligned}
$$

are all preserved under such limits.

Therefore we conclude that

$$
\frac{1-f(z) \overline{f(\zeta)}}{\prod_{j=1}^{d}\left(1-z_{j} \bar{\zeta}_{j}\right)}=K_{S}(z, \zeta)+L_{T}(z, \zeta)
$$

is a valid decomposition.

\section{Appendix: Reproducing Kernels}

We record a number of facts about reproducing kernels which we used above. We are sketchy since much of this is well-known. For general references see [2,3]. As before, $\mathcal{P}$ is the reproducing kernel for $L_{\mu}^{2}(B)$, where $d \mu=|p|^{-2} d \sigma$ and $B=\left\{\alpha \in \mathbb{Z}_{+}^{d}\right.$ : $\alpha \nsupseteq n\}$. (The details of $\mu$ and $B$ are by no means essential for what follows.) 
Lemma 7.1 ([3, Theorem 2.2]) A function $f: \mathbb{D}^{d} \rightarrow \mathbb{C}$ is in a reproducing kernel Hilbert function space $\mathcal{H}$ on $\mathbb{D}^{d}$ with kernel $K$ if and only if

$$
K(z, \zeta) \geq \epsilon f(z) \overline{f(\zeta)}
$$

for some $\epsilon>0$. The largest possible $\epsilon$ is equal to $\|f\|^{-2}$.

Lemma 7.2 Let $K$ be a positive semi-definite kernel on $\mathbb{D}^{d}$, and let $f$ be a finite linear combination of functions of the form $K_{\eta}(z):=K(z, \eta)$. Then, there is an $\epsilon>0$ such that

$$
K(z, \zeta) \geq \epsilon f(z) \overline{f(\zeta)}
$$

In the case of a single kernel function we can say

$$
K(z, \zeta) \geq \epsilon K_{\eta}(z) \overline{K_{\eta}(\zeta)}
$$

if and only if $1 \geq \epsilon K(\eta, \eta)$.

Proof Follows from Lemma 7.1.

Lemma 7.3 A positive semi-definite kernel $K$ satisfying $\mathcal{P} \geq K$ is a $\mathcal{P}$-kernel (as in Definition 1.4) if and only if for every function $f: \mathbb{D}^{d} \rightarrow \mathbb{C}$

$$
K(z, \zeta) \geq \epsilon f(z) \overline{f(\zeta)}
$$

implies

$$
K(z, \zeta) \geq \frac{f(z) \overline{f(\zeta)}}{\|f\|_{\mu}^{2}}
$$

in which case we necessarily have $\|f\|_{\mu}^{-2} \geq \epsilon$. In particular, $K(\zeta, \zeta)=\left\|K_{\zeta}\right\|_{\mu}^{2}$ holds for all $\zeta \in \mathbb{D}^{d}$ whenever $K$ is a $\mathcal{P}$-kernel. $\left(\right.$ Here $K_{\zeta}(z)=K(z, \zeta)$.)

Proof Follows from the definition of a $\mathcal{P}$-kernel and Lemma 7.1.

Lemma 7.4 Suppose $\mathcal{P} \geq K \geq 0$. Let $\mathcal{H}=\vee\left\{K_{\zeta}: \zeta \in \mathbb{D}^{d}\right\}$ be the closed span in $L_{\mu}^{2}(B)$ of the functions $K_{\zeta}(z)=K(z, \zeta)$, and let $L$ be the reproducing kernel for $\mathcal{H}$. Then, $L \geq K$.

Proof This essentially follows from Corollary 2.6 of [3].

Lemma 7.5 If $K$ is a reproducing kernel for a closed subspace of $L_{\mu}^{2}(B)$, then $K$ is a P-kernel.

Proof This follows from Lemmas 7.1 and 7.3 and the fact that the norm on a subspace is the same as the norm in the original space. 
Lemma 7.6 If a kernel $K$ with $\mathcal{P} \geq K$ is $z_{j}$-contractive, then

$$
K(z, \zeta) \geq \epsilon f(z) \overline{f(\zeta)}
$$

implies $f, z_{j} f \in L_{\mu}^{2}(B)$.

Proof By assumption, $\left(1-z_{j} \bar{\zeta}_{j}\right) K(z, \zeta) \geq 0$ and therefore

$$
\mathcal{P}(z, \zeta) \geq K(z, \zeta) \geq z_{j} \bar{\zeta}_{j} K(z, \zeta) \geq \epsilon z_{j} \bar{\zeta}_{j} f(z) \overline{f(\zeta)}
$$

which shows $z_{j} f \in L_{\mu}^{2}(B)$ (see Lemma 7.1).

Lemma 7.7 If $\mathcal{H}$ is a closed subspace of $L_{\mu}^{2}(B)$ and $\mathcal{H}$ is closed under multiplication by $z_{j}$, then the reproducing kernel for $\mathcal{H}$ is $z_{j}$-contractive.

See for example Corollary 2.37 of [2].

Acknowledgments I would like to thank the referee for a close reading.

Open Access This article is distributed under the terms of the Creative Commons Attribution Noncommercial License which permits any noncommercial use, distribution, and reproduction in any medium, provided the original author(s) and source are credited.

\section{References}

1. Agler, J.: Some Interpolation Theorems of Nevanlinna-Pick type. Unpublished manuscript, 1988

2. Agler, J., McCarthy, J.E.: Pick Interpolation and Hilbert Function Spaces. Graduate Studies in Mathematics, vol. 44. American Mathematical Society, Providence (2002)

3. Beatrous, F. Jr.., Burbea, J.: Positive-definiteness and its applications to interpolation problems for holomorphic functions. Trans. Am. Math. Soc. 284(1), 247-270 (1984)

4. Grinshpan, A., Kaliuzhnyi-Verbovetskyi, D.S., Vinnikov, V., Woerdeman, H.J.: Classes of tuples of commuting contractions satisfying the multivariable von Neumann inequality. J. Funct. Anal. 256(9), 3035-3054 (2009)

5. Rudin, W.: Function Theory in Polydiscs. W. A. Benjamin, New York (1969) 